\title{
Peut-on traduire une image ? Implications de la créativité dans la traduction de textes audiovisuels
}

\author{
Can images be translated? \\ Implications of creativity in the translation \\ of audiovisual texts
}

\author{
Julio de los Reyes Lozano \\ Universitat Jaume I \\ delosrey@uji.es \\ Frederic Chaume \\ Universitat Jaume I \\ chaume@uji.es
}

\begin{abstract}
Multimodality is an integral characteristic of Audiovisual Translation. Regardless of type and genre, audiovisual texts (i.e. film, TV series, videogame) are above all semiotic constructs comprising several signifying codes that operate simultaneously in the production of meaning. While audiences mechanically receive and interpret the information transmitted by each of these codes and signs, audiovisual translators must know their functioning and consider their possible impact on translation operations. In this context, images represent one of the major challenges to ensure coherence within audiovisual translation. This paper sets out to explore the main strategies used to explicit iconographic information in the target text. These practices typically involve, but are not limited to, the inclusion of linguistic signs related to the icon as well as the inclusion of signs belonging to other meaning codes, thanks to the possibilities that new technologies and digitalization now offer. Linked to the notions of transcreation and localization, these help target audiences fill gaps in the understanding of audiovisual texts, but also promote overtranslation. This may lead to an extreme domestication, a phenomenon in which foreign cultures would be erased. In this regard, we wonder if such practices will not assume a paternalistic approach to target audiences.
\end{abstract}

Keywords: audiovisual translation, multimodality, transcreation, iconography, overtranslation 


\section{LA LOCALISATION DE CONTENUS AUDIOVISUELS}

\section{LE TEXTE AUDIOVISUEL EN TANT QU'ENTITÉ VERBO-ICONIQUE}

D'après la définition classique de Kress et Van Leeuwen, selon laquelle un texte multimodal serait « any text whose meanings are realized through more than one semiotic code » (2006, p. 177), il est permis d'affirmer que la multimodalité fait partie intégrante de la traduction audiovisuelle. En effet, qu'il s'agisse d'un film, d'une publicité ou d'un jeu vidéo, le texte audiovisuel est avant tout un ensemble sémiotique où le sens est le produit du croisement et de l'interaction de plusieurs signes, structurés et organisés dans divers codes de signification et reproduits à travers, au moins, deux canaux de communication, le canal acoustique et le canal visuel (Chaume, 2012, p. 117) :

\author{
CANAL ACOUSTIQUE \\ Code linguistique \\ Code paralinguistique \\ Code musical \\ Code d'effets spéciaux \\ Code sonore
}

\author{
CANAL VISUEL \\ Code iconographique \\ Code photographique \\ Code de mobilité \\ Code de cadrage \\ Codes graphiques \\ Code de montage
}

Les problèmes spécifiques de la traduction audiovisuelle sont alors liés à - et peuvent s'expliquer par - l'interaction entre ces codes et les dialogues (le code linguistique). Bien que le langage cinématographique dispose d'autres codes de signification (par exemple, les codes technologiques), ceux-ci n'ont pas d'incidence significative sur les opérations de traduction et ne sont pas inclus dans la liste précédente (Casseti et Di Chio, 1991). Cependant, quelques films expérimentaux font recours à un troisième canal - le canal tactile - afin d'imiter les sensations perçues par les personnages à l'écran dans les fauteuils des spectateurs (par exemple, les vibrations ressenties lors d'un tremblement de terre). De même, dans certains jeux vidéo le canal tactile permet de contrôler les mouvements et les actions du joueur à travers les vibrations (des signes encodés dans le code « haptique ») (Mejías-Climent, 2019).

Ces spécificités ont un impact direct sur les opérations de traduction afin de garantir la suspension consentie de l'incrédulité propre aux œuvres audiovisuelles. Ainsi, le traducteur-adaptateur est censé prendre en considération l'interaction entre les signes employés par chaque code dans la production de sens, qui doivent être transférés à la culture d'arrivée, et respecter les contraintes associées sans que le spectateur sorte du film. Par exemple, dans le cas du doublage, lorsqu'un personnage parle en gros plan, on devrait faire attention aux codes de signification suivants : le code linguistique - les mots contenant des phonèmes bilabiaux ou des voyelles ouvertes; le 
code de mobilité - les personnages qui prononcent ces mots à l'écran ; et le code de cadrage - les gros plans ou les très gros plans.

Quant à l'étude de cette forme de traduction, une approche sémiotique et multimodale s'impose : l'interaction entre mots et images, la cohérence sémiotique de l'œuvre audiovisuelle ou la réitération fréquente de l'information à travers un ou plusieurs codes de signification se trouvent parmi les procédures d'analyse effectuées par les chercheurs en traduction audiovisuelle. Dans de nombreux cas, les images permettent de renforcer ou d'ancrer le sens spécifique d'un mot ou d'une phrase, alors que dans d'autres, c'est bien le contraire et les images s'opposent à ce sens-là pour provoquer une situation comique, de surprise ou de confusion. En règle générale, les traducteurs audiovisuels ont tendance à respecter l'image, qui est, en principe, intouchable ; ils cherchent donc à façonner les mots afin de maintenir le même rapport (d'ancrage, de réitération, de contradiction) entre les dialogues de la version originale et les images (et les autres signes visuels et acoustiques) de l'œuvre audiovisuelle.

\section{L'ADAPTATION DE CONTENUS AUDIOVISUELS, UN JEU D'ÉQUILIBRISTE EN PERMANENCE}

Le secteur professionnel de la traduction audiovisuelle est en évolution constante depuis quelques années, surtout à partir du « tournant technologique » (Díaz Cintas, 2013) qui a entraîné la numérisation tant de la production que de la postproduction de contenus audiovisuels et, par conséquent, des différents procédés d'adaptation (doublage, sous-titrage, voice-over, entre autres). Jusqu'à l'avènement de l' « accessible filmmaking » ou " production cinématographique accessible » (Romero-Fresco, 2013) ${ }^{1}$, ces procédés avaient toujours lieu une fois l'œuvre audiovisuelle terminée. Aujourd'hui encore, les responsables de l'adaptation (traducteur-adaptateur, chef de plateau) interviennent généralement lors des étapes de postproduction audio et vidéo, et c'est à ce moment-là que les différentes possibilités de manipulation du texte cible sont examinées dans le but d'obtenir un produit final cohérent.

En vue de cette cohérence, dans de nombreux cas, les dialogues doivent s'adapter aux images et aux autres signes sémiotiques du texte audiovisuel (Baumgarten, 2008), s'inscrivant souvent dans une stratégie de naturalisation et transformant l'œuvre étrangère en un produit cinématographique qui pourrait très bien appartenir à la culture d'arrivée. La traduction peut contribuer à l'édification du capital culturel audiovisuel, grâce à l'intégration dans la culture cible d'œuvres audiovisuelles canoniques appartenant aux cultures étrangères. Mais cet « habitus » peut évoluer - il est déjà en train de le faire - particulièrement au sein des communautés de spectateurs actifs («fansub-

\footnotetext{
${ }^{1}$ Cette pratique innovante encourage les professionnels à se focaliser sur la compréhension des spectateurs (plutôt que sur leur engagement) et à fournir les informations susceptibles d'être perdues en raison d'éventuelles déficiences sensorielles ou méconnaissance de la langue du film.
} 
bers » et « fandubbers »). Ces groupes montrent une préférence pour les traductions qui suivent une méthode de traduction littérale et adoptent des stratégies de dépaysement révélant l'origine géographique de l'œuvre audiovisuelle et conservant son parfum étranger, ce qui a été préconisé par certains traductologues (Venuti, 1995, p. 20).

Par ailleurs, on doit à ces communautés l'introduction de nouvelles pratiques telles que le sous-titrage créatif, qui ne suit pas les conventions formelles et professionnelles des sous-titres classiques et que nous aborderons dans la section suivante. Dans cet article, nous nous intéressons tout particulièrement à la traduction audiovisuelle créative qui a osé changer les règles du jeu en sens opposé, en se dirigeant vers une naturalisation extrême des adaptations. Il existe en effet des œuvres audiovisuelles traduites où non seulement les dialogues, mais aussi les effets sonores (Adachi, 2020), et surtout les images, ont été adaptés et naturalisés pour maximiser le degré d'acceptabilité du produit final.

La manipulation des images était liée jusqu'à récemment à la notion de censure, objet d'étude auquel les chercheurs en traduction audiovisuelle se sont beaucoup intéressés en tant que pratique méprisable et propre aux pays ayant des déficits démocratiques importants (voir par exemple Gutiérrez Lanza, 2002). Réservée à certains régimes dictatoriaux, la censure consiste à couper de l'œuvre audiovisuelle originale certaines scènes à caractère politique (ou religieuse, sexuelle, etc.) afin de ne pas montrer au public des valeurs morales différentes de celles prônées par ces régimes.

Cependant, on constate aujourd'hui des pratiques similaires dans des pays et sociétés qui, en principe, ne souffrent pas de ces carences. L'idée qui sous-tend cette manipulation des images dans la traduction serait motivée, non par des questions de censure politique ou religieuse, mais par des raisons purement économiques. Il s'est avéré qu'une meilleure acceptation de l'œuvre audiovisuelle doublée ou sous-titrée favorisait le succès commercial et augmentait les recettes. Par conséquent, en plus de faciliter la compréhension de l'œuvre, ce type de naturalisation cherche à plaire davantage et à mieux répondre aux attentes du public cible en reproduisant les valeurs de la société d'arrivée (des exemples illustratifs seront donnés dans la section suivante). Enfin, il ne faut pas oublier qu'un succès économique peut en cacher un autre : un film ou une série télévisée peuvent entraîner le développement d'univers transmédiatiques, via les différentes saisons d'une série ou des œuvres indépendantes - livres, jeux vidéo ou bandes dessinées, entre autres - appartenant au même univers narratif.

\section{VERS UNE TRADUCTION AUDIOVISUELLE CRÉATIVE}

La traduction audiovisuelle créative est un concept assez récent qui fait référence aux opérations d'adaptation de contenus audiovisuels qui, en plus de permettre l'accès à ces œuvres lorsqu'il existe des barrières linguistiques ou physiologiques, parviennent à provoquer une nouvelle expérience sensorielle chez le spectateur de la 
version traduite. Pour ce faire, elle est censée apporter des solutions imaginatives qui s'intègrent dans l'œuvre afin de devenir une contribution artistique en elle-même. Issue du domaine professionnel, la traduction audiovisuelle créative ne suit pas les conventions de l'adaptation telle que nous les connaissons aujourd'hui. De même, elle peut s'intégrer dans le texte filmique et faire partie du processus créatif - notamment à travers la production cinématographique accessible (Romero-Fresco, 2013), ou bien s'ajouter lors des étapes de postproduction.

Selon Bollettieri Bosinelli, les traducteurs audiovisuels pour le doublage « ha[ve] to be creative in order to be effective » (1994, p. 14). Compte tenu de la forte dépendance à l'image de la traduction audiovisuelle en général, nous oserions aller plus loin et élargir cet adage à la totalité des procédés, car ils demandent tous des solutions créatives de la part des adaptateurs, notamment dans le domaine de l'accessibilité. En effet, en plus de la production cinématographique accessible, on trouve des exemples de pratiques créatives d'audiodescription, de sous-titrage à destination des sourds et malentendants (Cómitre Narváez et Sedano Ruiz, 2019) et d'interprétation en langue des signes dans certains spectacles musicaux ${ }^{2}$. Dans les lignes qui suivent nous accordons toutefois une attention particulière aux procédés les plus répandus, le doublage et le sous-titrage.

\section{LE DOUBLAGE CRÉATIF}

En tant que forme de traduction, le doublage consiste à remplacer, de façon synchrone avec l'image, les voix originales par d'autres dans la langue cible. En raison de la liberté d'action qu'offre cette spécificité, Pruys (2009) considère la version doublée d'un film comme indépendante et autonome, tout en soulignant que le texte source et le texte cible sont rarement équivalents en termes de forme et de contenus. Il s'agirait plutôt de deux variations du même thème, raison pour laquelle cette forme de « traduction totale » (Cary, 1960) a également été qualifiée de « transadaptation »ou «re-creation» (Leppihalme, 1997, p. 100).

Le terme « transcréation » est également employé pour se référer aux opérations transformatives de traduction qu'on peut parfois rencontrer dans le doublage (Zanotti, 2014, p. 110). Utilisée principalement dans le domaine de la publicité, cette pratique suit le principe de " glocalisation »- combinaison de globalisation et localisation - selon lequel un produit global est adapté à chacune des cultures à laquelle il s'adresse. Dans ce contexte, nous entendons la « transcréation » comme un type de traduction audiovisuelle enrichie, conséquence directe de la numérisation, car la technologie permet aujourd'hui de manipuler les images plus facilement qu'auparavant. À cet égard, Chaume (2016) propose le terme « enhanced dubbing » ou « doublage enrichi » pour désigner les opérations de traduction et d'adaptation pour le doublage incluant la manipulation des images.

${ }^{2}$ https://www.rocksinsubtitulos.uk/noticia/rozalen-y-otros-artistas-que-usan-lengua-de-signos 
Cette manipulation apparaît principalement lorsque des messages écrits en langue source sont visibles à l'écran, ce qui arrive très souvent sous forme de panneaux ou d'affiches. En fonction de son importance dans l'œuvre, l'information de ces messages peut être explicitée par le biais d'une traduction. Pour cela, Chaume distingue globalement les stratégies suivantes, spécifiques de ce procédé d'adaptation (2012, p. 117) : enregistrer le texte en voix off, le sous-titrer, ou bien éditer les images pour insérer le texte cible. Si les deux premières sont les plus répandues, car l'image reste inchangée et le processus est donc moins problématique, c'est précisément la troisième qui nous intéresse du point de vue du doublage créatif, en raison de la manipulation des images lors du processus d'adaptation.

La plupart du temps, la motivation de ce type de manipulation est purement esthétique et vise à préserver les qualités artistiques de l'œuvre audiovisuelle, comme c'est le cas de certains films d'animation, où les scènes montrant des messages écrits sont « redessinées » pendant les étapes de production pour insérer la traduction en langue cible (Iglesias, 2009, p. 384). À titre d'exemple, le film Là-Haut [Up] (Docter et Peterson, 2009) construit une séquence essentielle autour d'un message écrit : vers la fin
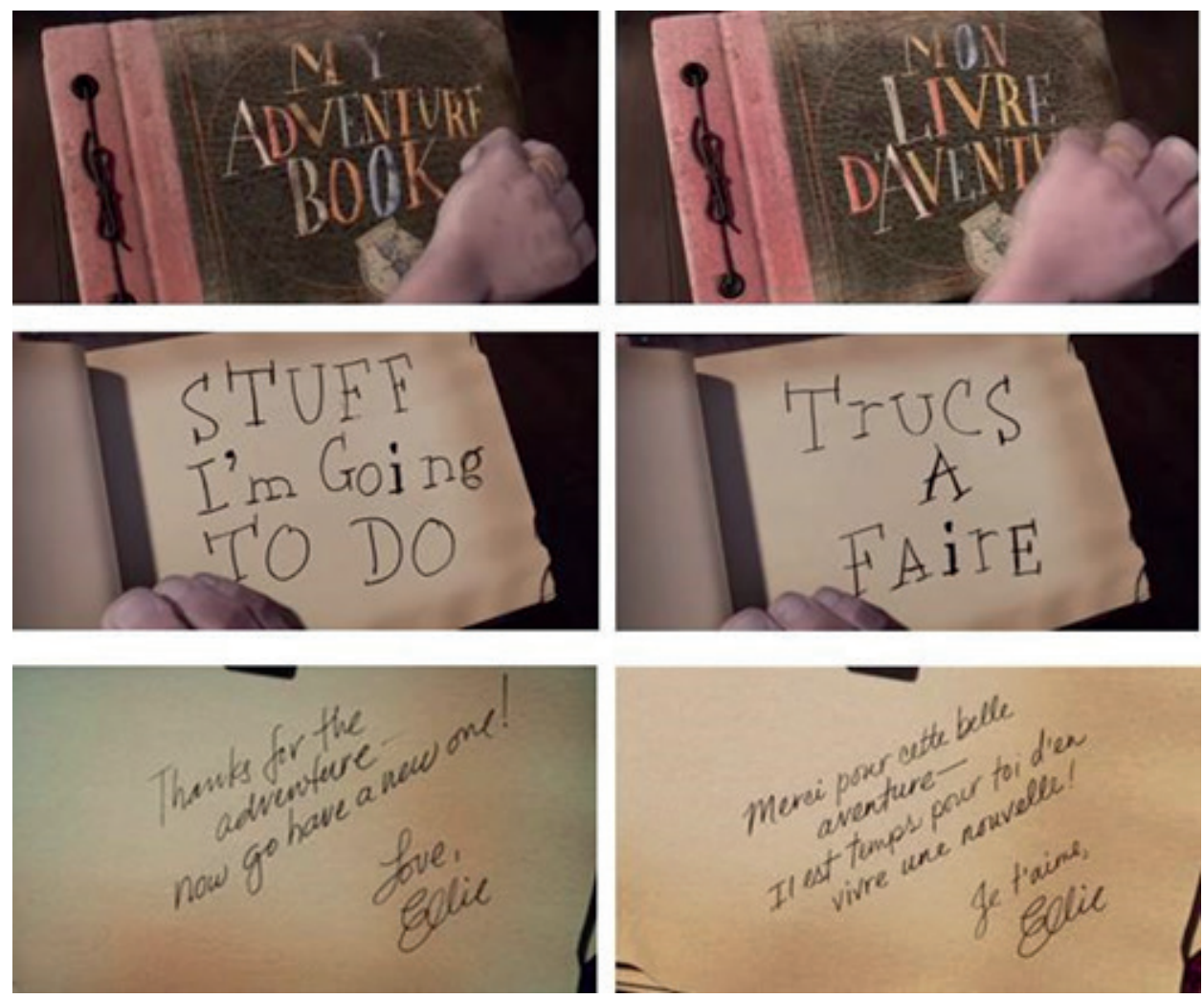

Fig. 1. Photogrammes des versions originale et française de Là-haut [Up] (Docter et Peterson, 2009) 
du film, le vieil homme protagoniste est en train de feuilleter le livre d'aventures de son épouse décédée et, parmi les nombreux souvenirs d'enfance, découvre un message inattendu qui sera le déclencheur de la résolution de l'histoire (voir Image 1 ci-dessus). À défaut de dialogue, l'accent est mis sur la musique, qui renforce le caractère intimiste de la séquence. En ce qui concerne la version traduite, le « redessinage » évite que le spectateur sorte du film dans un moment particulièrement poignant, grâce à l'absence de distractions visuelles (sous-titres) ou sonores (enregistrement en voix off).

Mais la manipulation des messages écrits peut également influencer quelques aspects culturels. C'est ce qui arrive dans le film Captain America : Le Soldat de l'hiver [Captain America: The Winter Soldier] (Russo et Russo, 2014), lorsque le superhéros cherche à mémoriser une liste d'événements historiques et culturels qu'il a manqués lorsqu'il était congelé. Ce qui est drôle, c'est que la liste diffère selon la zone géographique, comme le montre le site The Idealist ${ }^{3}$, qui recueille les différences constatées dans les versions russe, britannique, française, mexicaine, italienne et espagnole du film. Dans une perspective évidente de «glocalisation », on voit comment les intérêts de Captain America perdent le côté politique dans les versions française et britannique et renforcent le côté culturel et sportif. Par exemple, la référence au Mur de Berlin - ainsi que son halo d'humour ( « up + down ») - est remplacée par The Beatles au Royaume-Uni et le comédien Coluche en France ${ }^{4}$. En revanche, les spectateurs russes et espagnols ont droit à des moments politiques, mais plus proches des sociétés d'arrivée (Dissolution de l'Union Soviétique et Constitution de 1978 respectivement). Au-delà de cette naturalisation extrême, notre attention est attirée par le fait que la liste est écrite en anglais dans toutes les versions.

Un autre exemple de « transcréation » ou « doublage enrichi » est l'adaptation du célèbre dessin animé japonais Doraemon (Kusuba, 2005-) à la culture américaine, où certaines scènes ont été redessinées pour les rendre plus attractives ou prétendument tolérables aux enfants des États-Unis, le public cible. Parmi ces opérations sémiotiques, il a été observé que les billets de yen originaux ont été substitués par des dollars, les baguettes pour manger par des fourchettes, et que les notes des examens visibles à l'écran ont aussi été adaptées (Chaume, 2016). En outre, les larmes et le sang de certaines scènes ont disparu de la version doublée (voir image ci-dessous).

Ce type d'adaptation existe aussi dans l'autre sens et, par exemple, certains plans de la version japonaise du film Vice-versa [Inside Out] (Docter et Del Carmen, 2015) ont été redessinés : afin de montrer l'aversion de l'enfant protagoniste envers la nourriture, le réalisateur Docter explique que le brocoli a été remplacé par des poivrons verts car, apparemment, cet aliment est considéré comme dégoûtant par les enfants

\footnotetext{
${ }^{3} \mathrm{http}: / /$ www.theidealist.es/la-lista-del-capitan-america-que-cambia-segun-el-pais-donde-se-vea/

${ }^{4} \mathrm{Ce}$ choix affecte également la dimension interne du personnage, ce qui est assez surprenant du fait que l'engagement et la défense des valeurs démocratiques est l'un des signes distinctifs de Captain America (Dorvidal, 2003, p. 258).
} 


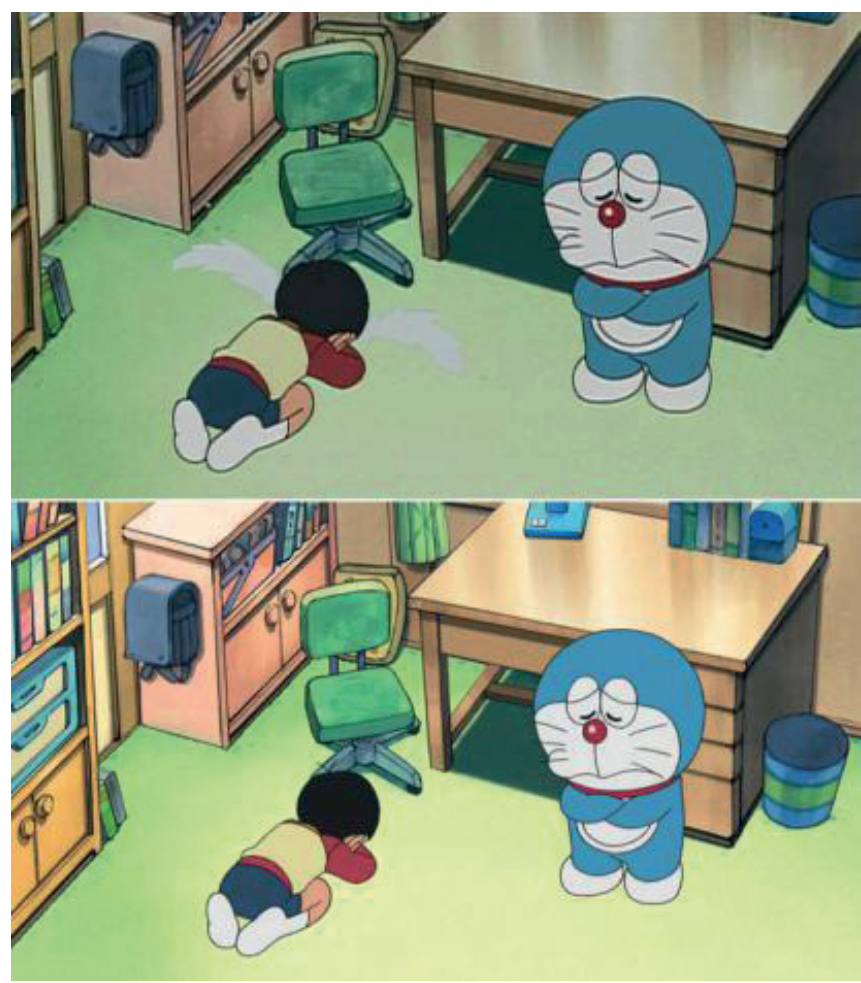

Fig. 2. Photogrammes des versions originale et américaine de Doraemon (Kozo Kusuba, 2005-)

japonais ${ }^{5}$. Mais à en croire Docter, celui-ci n'est pas le seul exemple de modification iconographique subie par les versions internationales de ce film et jusqu'à quarante-cinq plans ont été localisés, tel que le hockey sur glace de la version originale qui a été substitué par du football dans la version britannique. De telles modifications auraient pour but de chercher l'implication des spectateurs en s'adaptant « au plus près du profil de leur public cible à l'étranger, dont le marché est alors segmenté culturellement, dans une stratégie de "traduction" extensive » (Brisset, 2019, p. 26).

\section{LE SOUS-TITRAGE CRÉATIF}

Comme il a été évoqué précédemment, les caractéristiques du sous-titrage créatif diffèrent des conventions formelles et professionnelles des sous-titres classiques. Aujourd'hui, on trouve beaucoup de ces pratiques créatives dans ce que Díaz Cintas appelle « cybersubtitles », un terme qui regroupe les différents types de sous-titres

\footnotetext{
${ }^{5}$ https://slate.com/culture/2015/07/inside-out-director-pete-docter-explains-why-pixar-re-animatedcertain-scenes-like-the-broccoli-scene-for-international-audiences.html
} 
réalisés par des utilisateurs « outside the commercial imperatives that regulate professional practice » $(2018$, p. 141) et qui contournent les normes du sous-titrage avec créativité. Ces formules comprennent l'application de techniques de dialogue, l'augmentation du nombre de lignes par sous-titre, la diversification des couleurs ou le recours à des sous-titres cumulatifs et des bulles pop-up pour donner des explications à la manière des notes en bas de page.

Cependant, la traduction audiovisuelle créative ne se limite pas aux sous-titres faits par des amateurs et s'étend aux professionnels de la traduction et du cinéma. En effet, certains réalisateurs vont de plus en plus dans cette direction ${ }^{6}$ et McClarty se réfère au succès de Slumdog Millionaire (Boyle, 2008) et d'autres films multilingues pour démontrer l'utilité des sous-titres créatifs d'un point de vue esthétique (2012, p. 149). À partir de l'étude de plusieurs exemples de films récents, l'auteure suggère la mise en œuvre, dans le sous-titrage, de pratiques créatives propres au cinéma, telles que l'utilisation de couleurs, de polices, de styles et d'emplacements différents, tout comme d'effets spéciaux (2012, p. 150).

L'essor du cinéma 3D au début des années 2010 a favorisé l'application de certaines de ces pratiques dans la traduction des films en relief. Dans leur analyse de la version sous-titrée en français d'Avatar (Cameron, 2009), Baldo de Brébisson et Hamus-Vallée (2021) ont observé que les sous-titres « flottants » participent, eux aussi, aux effets de la 3D. Ainsi, par exemple l'emplacement des sous-titres sur l'axe Z propre à la 3D et le décalage existant par rapport à l'axe classique X-Y crée un effet de jaillissement ou de profondeur. De même, les auteures affirment que l'espace accordé aux caractères varie selon la place choisie sur l'écran, ce qui comporte le plus souvent une contrainte traductionnelle du fait de la réduction de l'axe $\mathrm{X}$.

La présence de sous-titres créatifs est aussi habituelle dans certaines émissions d'infodivertissement, lorsque l'action des reportages et des chroniques se déroule dans une zone géographique avec une langue différente à celle de l'émission. C'est le cas de Quotidien, diffusée quotidiennement sur la chaîne française TMC et présentée par Yann Barthès? . Cette émission aborde habituellement des sujets internationaux, notamment américains, et utilise le sous-titrage créatif pour la traduction des interventions en langue étrangère. Qu'il s'agisse d'informations à caractère politique (élections) ou culturel (célébrités), les traits distinctifs des sous-titres de Quotidien sont le changement constant d'emplacement, l'affichage moyennant un «boxing » de couleurs différentes, ou encore l'adaptation de la taille et du format des sous-titres en fonction des interventions (voir images ci-dessous).

En outre, les sous-titres de Quotidien ont tendance à se coller aux discours et s'affichent à l'écran au fur et à mesure que les intervenants parlent (voir image 3). De même, ils cherchent à reproduire les gestes paralinguistiques, voire exagérer les

${ }^{6}$ Fox (2017) montre plusieurs exemples de films et de séries télévisées multilingues qui intègrent des sous-titres dans la composition de l'œuvre originale.

${ }^{7} \mathrm{https}: / / \mathrm{www} . \mathrm{tf1}$.fr/tmc/quotidien-avec-yann-barthes 
exclamations ou interjections (voir image 4). À cet égard, il arrive aussi que certains mots soient affichés en majuscules et en plus gros que les autres selon l'intonation du locuteur, comme dans l'exemple de l'image 3. Enfin, les sous-titres contiennent souvent des effets spéciaux et on a droit à des sous-titres qui volent, brillent ou tremblent, entre autres effets, afin d'accompagner les mouvements des personnages ou de souligner l'importance d'un mot concret (voir image 5).

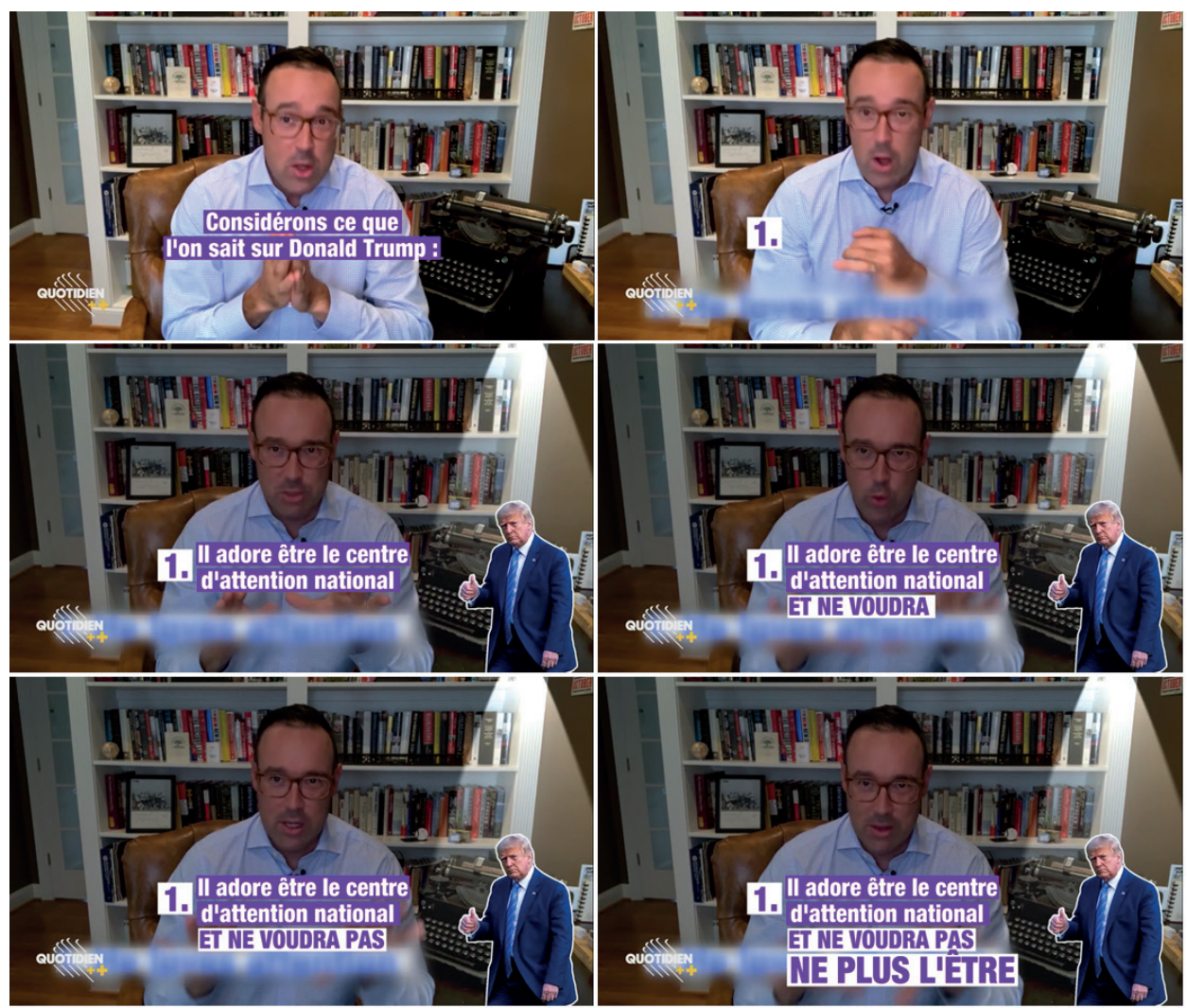

Fig. 3. Exemple de sous-titres collés aux interventions dans Quotidien
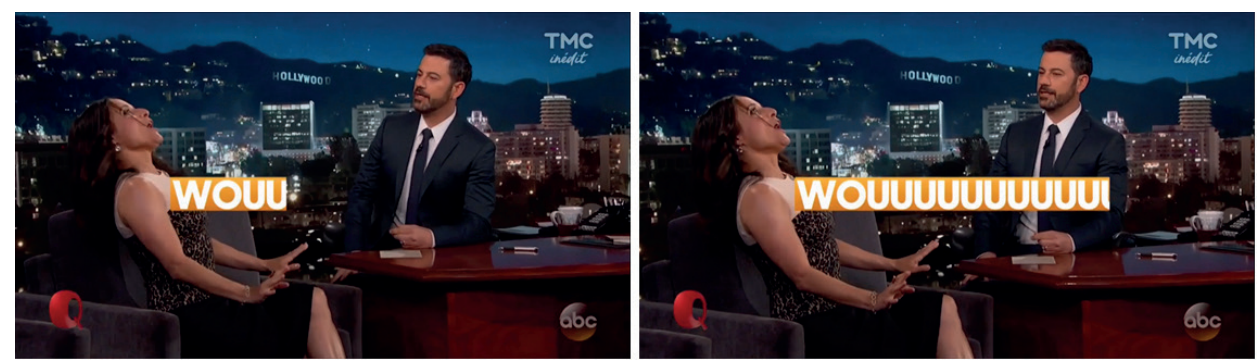

Fig. 4. Exemple de sous-titre qui reproduit les gestes paralinguistiques dans Quotidien 


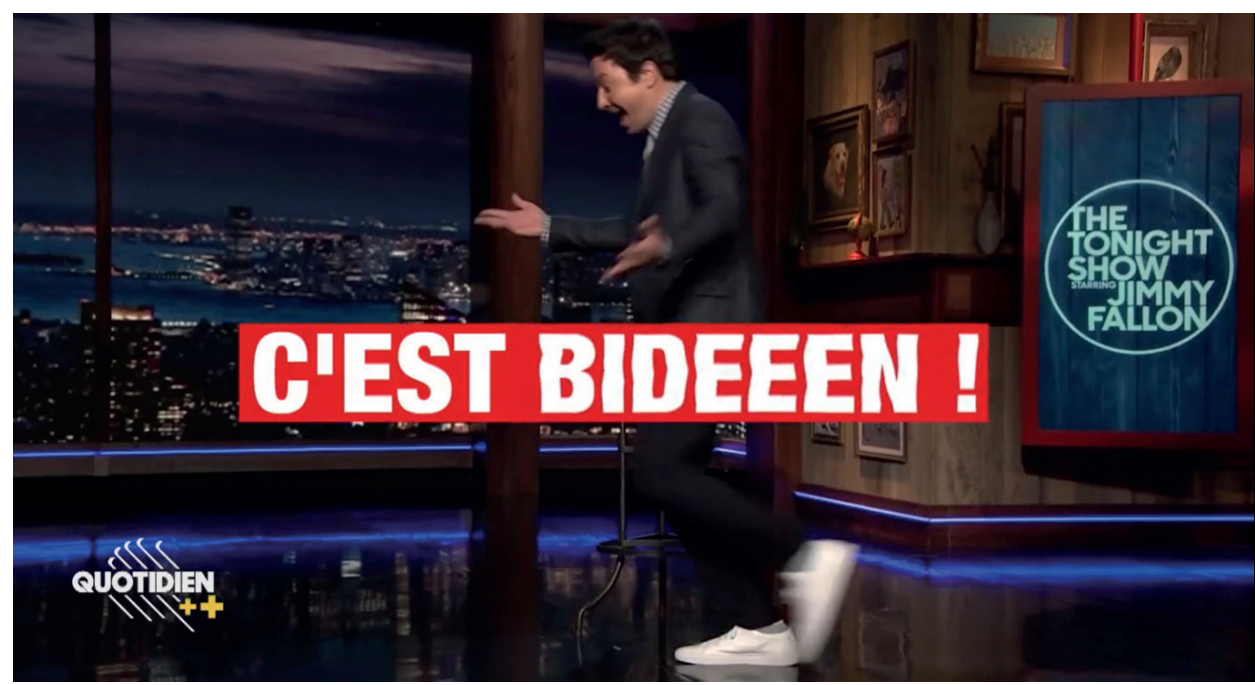

Fig. 5. Exemple de sous-titre qui tremble dans Quotidien

Reste à connaître l'avis du public à propos de ces pratiques créatives, dont l'étude de réception réalisé par Fox (2018) représente une première étape. Cette auteure a mené à bien une expérience utilisant des questionnaires et des techniques d'oculométrie pour évaluer la réception des sous-titres créatifs auprès d'un groupe de spectateurs anglais et allemands. Les résultats montrent, d'une part, que les participants ont qualifié les sous-titres créatifs comme plus esthétiques comparés aux sous-titres traditionnels. D'autre part, il a été observé que ce type de sous-titres peut réduire le temps de lecture et motiver le spectateur de la version sous-titrée à retourner plus rapidement au point central de l'image, la durée de fixation étant plus proche de celle des spectateurs de la version originale. Finalement, les participants sembleraient avoir expérimenté une prise d'information plus complète (Fox, 2018, p. 198).

\section{POUR ALLER UN PEU PLUS LOIN : LES LIMITES DE LA TRADUCTION AUDIOVISUELLE}

Si nous allons un peu plus loin, nous pouvons contourner les limites du concept de traduction à travers deux exemples de transferts textuels où les images sont en quelque sorte « traduites » : les adaptations et les remakes de films et de séries télévisées. Dans ces cas-là, il est facile de trouver des traductions de tous les codes de signification présents dans le texte audiovisuel ${ }^{8}$ : de la traduction ou transposition

${ }^{8} \mathrm{Il}$ est intéressant de noter que le terme « adaptation » est utilisé dans les pays francophones pour se référer aux différentes versions d'une œuvre dans d'autres domaines artistiques (roman ou bande dessinée dans un film, par exemple), mais aussi comme synonyme de traduction audiovisuelle. 
des dialogues à la «traduction » des décors, des costumes, des référents culturels et des coutumes, entre autres. Parmi les nombreux exemples où l'on peut observer ces transferts sémiotiques, nous pouvons citer la série espagnole Cuéntame cómo pasó (Bernardeau, 2001-) et ses adaptations italienne (Raccontami), portugaise (Conta-me

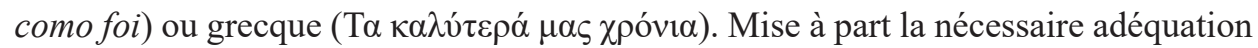
culturelle au cas par cas, toutes ces adaptations suivent les expériences d'une famille de classe moyenne, racontent les mêmes intrigues et reproduisent des dialogues similaires. Les similitudes avec la série originale vont des noms des personnages aux titres des épisodes et même au montage du générique d'ouverture.

Les remakes transnationaux pour leur part sont considérés non pas « a unitary oneway process of cultural homogenisation but rather as an interstitial process through which cultures borrow from and interact with one another » (Perkins et Verevis, 2015, p. 677). Au final, les adaptations et les remakes d'une œuvre sont considérés comme des emprunts culturels à l'échelle globale, et acquièrent un nouveau statut qui va au-delà de la logique commerciale qui anime leur création, constituant un exemple de « traduction culturelle».

\section{CONCLUSION}

La traduction audiovisuelle créative comprend toutes ces pratiques qui, d'une part, permettent l'accès linguistique et culturel à des contenus audiovisuels, et d'autre part, présentent des contributions artistiques en elles-mêmes et provoquent de nouvelles expériences sensorielles chez le spectateur. Dans l'ère du « cybersubtitling » et du « cyberdubbing » (Díaz Cintas, 2018), ces opérations ne suivent pas les conventions professionnelles classiques de la traduction audiovisuelle. En revanche, il y a bien des exemples de créativité à suivre - ou à éviter, selon la perspective que l'on adopte - dans des œuvres audiovisuelles canoniques de tous types et genres, allant des films d'auteur ou grand public (d'animation ou en prise de vue réelle) aux séries télévisées de qualité ou aux émissions d'infodivertissement. Comme nous l'avons vu, l'utilisation de stratégies créatives a augmenté considérablement dans les doublages et les sous-titrages commerciaux. Cela permet d'attirer l'attention sur le traducteur et les autres agents intervenant dans le processus d'adaptation, de les rendre plus visibles et de leur accorder un rôle prépondérant, comme le réclamait le « tournant du traducteur».

Dans un monde de plus en plus numérique, la traduction audiovisuelle créative a acquis une nouvelle signification suite à l'exploration de techniques innovantes, et particulièrement aux possibilités offertes par les nouvelles technologies. Les exemples montrés peuvent probablement être accusés de favoriser une naturalisation extrême, et donc d'effacer les cultures étrangères, mais aussi de comporter une approche paternaliste envers les destinataires. Cependant, les nouvelles technologies encouragent 
la participation active des nouveaux publics («fansubbers », « fandubbers ») dans le processus de traduction, et les pratiques « amateurs » qui s'éloignent des conventions professionnelles trouvent peu à peu leur place dans les doublages et les sous-titrages commerciaux. Les traducteurs-adaptateurs créatifs ont su trouver les moyens pour apporter leur touche esthétique par le biais de la transgression des formats, défiant les conventions établies, rajoutant ou omettant des informations et, ce faisant, prenant parti. 


\section{BIBLIOGRAPHIE}

Adachi, R. (2020). Dubbing of Sound in the Samurai Movie Love and Honor. InTRAlinea, 22. Retrieved from : http://www.intralinea.org/archive/article/2522.

Adami, E. \& Ramos Pinto, S. (2020). Meaning-(re)making in a world of untranslated signs: towards a research agenda on multimodality, culture, and translation. In M. Boria, A. Carreres, M. Noriega-Sánchez, M. Tomalin (eds.), Translation and Multimodality. Beyond Words (pp. 71-93). London $\&$ New York : Routledge.

Baldo de Brébisson, S. \& Hamus-Vallée, R. (2021). Le sous-titrage relief d'Avatar, la transgression comme norme. In S. Genty, S. Baldo de Brébisson, J. De los Reyes Lozano (eds.), La traduction audiovisuelle : normes, transgressions et nouveaux défis professionnels (pp.19-36). Montpellier : L'Entretemps.

Baumgarten, N. (2008). Yeah: That's it! Verbal Reference to Visual Information in Film Texts and Film Translations. Meta, 53 (1), 6-25. DOI : 10.7202/017971ar.

Bollettieri Bosinelli, R.M. (1994). Film dubbing: Linguistic and cultural issues. Il traduttore nuovo, 42 (1), $7-28$.

Brisset, F. (2019). Le doublage audiovisuel, à l'épreuve du cosmopolitisme et de l'interculturalité. In M.M. Ogea Pozo \& F. Rodríguez Rodríguez (eds.), Insights into audiovisual and comic translation. Changing perspectives on films, comics and videogames (pp. 11-30). Córdoba : UCOPress.

Cary, E. (1960). La Traduction Totale. Babel, 6 (3), 110-115. DOI : 10.1075/babel.6.3.02car.

Casetti, F. \& Di Chio, F. (1991). Cómo analizar un film. Barcelona : Paidós.

Chaume, F. (2004). Film Studies and Translation Studies: Two Disciplines at Stake in Audiovisual Translation. Meta, 49 (1), 12-24. DOI : 10.7202/009016ar.

Chaume, F. (2016). Audiovisual Translation Trends: Growing Diversity, Choice, and Enhanced Localization. In A. Esser, I.R. Smith \& M.Á. Bernal-Merino (eds.), Media Across Borders. Localizing TV, Film and Video Games (pp. 68-84). New York : Routledge.

Chaume, F. (2018). Is audiovisual translation putting the concept of translation up against the ropes? JoSTrans - The Journal of Specialised Translation, 30, 84-104. Retrieved from : https://jostrans.org.

Cómitre Narváez, I. \& Sedano Ruiz, E. (2019). Sous-titrage créatif pour enfants sourds et malentendants : les contes au cinéma, Palimpsestes, 32, 203-222. DOI : 10.4000/palimpsestes.3635.

Díaz Cintas, J. (2013). The Technology Turn in Subtitling. In M. Thelen \& B. Lewandowska-Tomaszczyk (eds.), Translation and meaning. Part 9 (pp. 119-132). Maastricht : Zuyd University of Applied Sciences.

Díaz Cintas, J. (2018). 'Subtitling's a carnival': New practices in cyberspace. JoSTrans - The Journal of Specialised Translation, 30, 127-149. Retrieved from : https://jostrans.org.

Dorvidal, J. (2003). Super-héros des Comics-books américains. In J. Vion-Dury \& P. Brunel (eds.), Dictionnaire des mythes du fantastique (pp. 254-260). Limoges : Presses Universitaires de Limoges.

Fox, W. (2017). A proposed set of placement strategies for integrated titles based on an analysis of existing strategies in commercial films. inTRAlinea, 19. Retrieved from : http://www.intralinea.org/specials/ $\operatorname{article} / 2250$.

Fox, W. (2018). Can integrated titles improve the viewing experience? Investigating the impact of subtitling on the reception and enjoyment of film using eye tracking and questionnaire data. Berlin : Language Science Press. DOI : 10.5281/zenodo.1180721.

Gutiérrez Lanza, C. (2002). Spanish Film Translation and Cultural Patronage: The Filtering and Manipulation of Imported Material during Franco's Dictatorship. In M. Tymoczko \& E. Gentzler (eds.), Translation and Power (pp. 141-159). Amherst \& Boston : University of Massachusetts Press.

Iglesias, L.A. (2009). Los doblajes en español de los clásicos Disney (unpublished doctoral disertation). Universidad de Salamanca, Spain. 
Kress, G. \& Van Leeuwen, T. (2006). Reading Images: The Grammar of Visual Design (Revised edition). London \& New York : Routledge.

Leppihalme, R. (1997). Cultural Bumps: An Empirical Approach to the Translation of Allusions. Clevedon : Multilingual Matters Ltd.

McClarty, R. (2012). Towards a Multidisciplinary Approach in Creative Subtitling. MonTI, 4, 133-155. DOI : 10.6035/MonTI.2012.4.6.

Mejías-Climent, L. (2019). La sincronización en el doblaje de videojuegos : análisis empírico y descriptivo (unpublished doctoral disertation). Universitat Jaume I, Spain.

Perkins, C. \& Verevis, C. (2015). Transnational television remakes. Continuum: Journal of Media \& Cultural Studies, 29 (5), 677-683. DOI : 10.1080/10304312.2015.1068729.

Pruys, G.M. (2009). Die Rhetorik der Filmsynchronisation. Köln : Eigenverlag.

Romero-Fresco, P. (2013). Accessible filmmaking: Joining the dots between audiovisual translation, accessibility and filmmaking. JoSTrans - The Journal of Specialised Translation, 20, 201-223. Retrieved from : https://jostrans.org.

Venuti, L. (1995). The Translator's Invisibility. A History of Translation. Oxon : Routledge.

Zanotti, S. (2014). Translation and transcreation in the dubbing process. A genetic approach. Cultus, 7, 109-134. Retrieved from : https://cultusjournal.com. 